\title{
Gravity wave transmission diagram
}

\author{
Y. Tomikawa ${ }^{1,2}$ \\ ${ }^{1}$ National Institute of Polar Research, Tokyo, Japan \\ ${ }^{2}$ SOKENDAI (The Graduate University for Advanced Studies), Tokyo, Japan
}

Correspondence to: Y. Tomikawa (tomikawa@nipr.ac.jp)

Received: 5 September 2015 - Revised: 24 November 2015 - Accepted: 24 November 2015 - Published: 1 December 2015

\begin{abstract}
A new method of obtaining power spectral distribution of gravity waves as a function of ground-based horizontal phase speed and propagation direction from airglow observations has recently been proposed. To explain gravity wave power spectrum anisotropy, a new gravity wave transmission diagram was developed in this study. Gravity wave transmissivity depends on the existence of critical and turning levels for waves that are determined by background horizontal wind distributions. Gravity wave transmission diagrams for different horizontal wavelengths in simple background horizontal winds with constant vertical shear indicate that the effects of the turning level reflection are significant and strongly dependent on the horizontal wavelength.
\end{abstract}

Keywords. Atmospheric composition and structure (airglow and aurora) - meteorology and atmospheric dynamics (waves and tides)

\section{Introduction}

An airglow imager is a unique instrument that can detect the horizontal structures of gravity waves around the mesopause region with high temporal and horizontal resolutions (i.e. $\sim 1$ min and $\sim 1 \mathrm{~km}$, respectively). Nearly monochromatic gravity waves have been extracted from airglow data by visual inspection and examined in previous studies (e.g. Taylor et al., 1993; Nakamura et al., 1999; Hecht et al., 2001). However, this method is time consuming and relies upon the ability of data analysts to detect the wave-like structures from the airglow data. Thus, methods that are more objective and universally applicable for describing the characteristics of gravity waves in airglow images are required.

Matsuda et al. (2014) proposed a new method (hereafter, Matsuda's method) to obtain a power spectral distribution of gravity waves as a function of ground-based horizontal phase speed and propagation direction from airglow data. They applied a three-dimensional (i.e. zonal, meridional and temporal) fast Fourier transform (3-D FFT) to the airglow emission intensity data at Syowa Station, Antarctica $\left(69^{\circ} \mathrm{S}, 40^{\circ} \mathrm{E}\right)$, af ter some corrections and converted the obtained 3-D power spectrum into the polar plot with ground-based horizontal phase speed as the radius and propagation direction as the polar angle. The peaks in the obtained power spectral distributions closely corresponded to the gravity waves obtained using a conventional event analysis based on visual inspection. Matsuda's new method enabled rapid parallel analysis of airglow data with different temporal and spatial resolutions, which is highly beneficial for the comparison of gravity wave statistics between different locations.

Gravity waves observed by airglow imagers generally have short horizontal wavelengths (i.e. $<100 \mathrm{~km}$ ) and propagation times shorter than a few hours from the troposphere up to the mesopause region (e.g. Stockwell and Lowe, 2001). Thus, it is often assumed that the propagation times and horizontal propagation distances of gravity waves during their vertical propagation from a source region up to the mesopause are small such that temporal and horizontal changes of the background horizontal winds can be ignored. Based on this assumption, using a vertical distribution for the background horizontal winds, Taylor et al. (1993) introduced a critical level blocking diagram that represents a restricted region of gravity wave propagation from the source region up to the mesopause due to critical level filtering. Gravity waves with ground-based horizontal phase speeds and propagation directions within this restricted region would encounter the critical level during their upward propagation and thus would be unable to reach the mesopause region. Such blocking diagrams enable assessment of whether the power spectrum anisotropy is due to critical level filtering. 
Gravity wave reflection at a turning level has never been considered in previous studies using airglow data. This is probably because the horizontal wavelength of a gravity wave is required to determine its turning level in addition to the background horizontal winds. However, the horizontal wavelength can be obtained using the 3-D FFT in Matsuda's method, which makes it possible to seek the turning level of the wave. A gravity wave transmission diagram, which represents a restricted region of gravity wave propagation due to critical level filtering and turning level reflection, is presented in this article. The relative effects of critical level filtering and turning level reflection on the vertical propagation of gravity waves from their source region to the mesopause are also demonstrated. The effects of additional critical level filtering, which would occur before the intrinsic horizontal phase speed of the wave becomes zero, are also discussed.

The theoretical backgrounds of the critical and turning levels are described in Sect. 2. Section 3 shows how a restricted region of gravity wave propagation due to critical level filtering and turning level reflection could change in relation to the horizontal wavelength of a wave under simple background horizontal wind conditions. The applicability of the gravity wave transmission diagram is discussed in Sect. 4. Concluding remarks are given in Sect. 5.

\section{Theoretical background}

\subsection{Critical level filtering}

Vertically propagating gravity waves are absorbed at a critical level at which the background horizontal wind speed component parallel to a horizontal wavenumber vector is equal to the ground-based horizontal phase speed of the wave (Booker and Bretherton, 1967). In other words, the intrinsic horizontal phase speed of the wave becomes zero at the critical level. This intrinsic horizontal phase speed is given by

$\hat{c}_{\mathrm{h}}=c_{\mathrm{h}}-(u \cos \theta+v \sin \theta)$,

where $c_{\mathrm{h}}$ and $\hat{c}_{\mathrm{h}}$ are the ground-based and intrinsic horizontal phase speeds, respectively, $u$ and $v$ are the background zonal and meridional winds, respectively, and $\theta$ is the direction of the horizontal wavenumber vector (and ground-based horizontal phase speed) (i.e. degrees anticlockwise from eastward). Thus, $\hat{c}_{\mathrm{h}}=0$ at the critical level. It should be noted that the intrinsic phase speed can be in the opposite direction to the ground-based phase speed when the background wind along the horizontal wavenumber vector is sufficiently strong. In short, $\hat{c}_{\mathrm{h}}$ can take either sign, while $c_{\mathrm{h}}$ is positive definite. The convention, where $c_{\mathrm{h}}$ is positive definite, simplifies the definition of source waves whose transmission is to be considered and eases the interpretation of the transmission diagrams. However, it is noted that this convention differs to that used in Fritts and Alexander (2003) where $\hat{\omega}$ (and thus $\hat{c}_{\mathrm{h}}$ ) is defined as positive definite. Stationary gravity waves (i.e. mountain waves), for which the direction of the groundbased phase speed cannot be determined, require an alternative treatment (Dunkerton and Butchart, 1984; Whiteway and Duck, 1996).

When a vertical distribution of background horizontal winds is given, Eq. (1) can be used to determine the height of the critical level where the intrinsic horizontal phase speed changes sign with height. Although Taylor et al. (1993) defined the height of the critical level to be where the intrinsic horizontal phase speed changes from positive to negative with height, their definition excludes gravity waves for which the directions of the ground-based and intrinsic horizontal phase speeds are opposite (e.g. Thomas et al., 1999). The effects of such a difference in the definition of the critical level are discussed in Sect. 3.

\subsection{Additional critical level filtering}

An intrinsic horizontal phase speed of zero, which is generally used as the definition of the critical level, corresponds to a zero intrinsic wave frequency. However, from the dispersion relation of inertia-gravity waves given by

$m^{2}=\frac{N^{2}-k_{\mathrm{h}}^{2} \hat{c}_{\mathrm{h}}^{2}}{\hat{c}_{\mathrm{h}}^{2}-f^{2} / k_{\mathrm{h}}^{2}}-\frac{1}{4 H^{2}}$

(cf. Fritts and Alexander, 2003), where $N$ and $f$ are buoyancy and inertial frequencies, respectively, $k_{\mathrm{h}}$ and $m$ are the horizontal and vertical wavenumbers of the wave, respectively, and $H$ is a scale height, $m^{2} \rightarrow \infty$ at $\hat{c}_{\mathrm{h}}= \pm \frac{f}{k_{\mathrm{h}}}$. Note that $k_{\mathrm{h}}$ is positive definite. Thus, the lowest intrinsic frequency of a vertically propagating gravity wave is the inertial frequency in a rotating fluid. A critical level of a gravity wave in a rotating fluid exists at the height at which $\hat{c}_{\mathrm{h}}= \pm \frac{f}{k_{\mathrm{h}}}$ and provides more gravity wave filtering than does the critical level defined by $\hat{c}_{\mathrm{h}}=0$.

\subsection{Turning level reflection}

In linear theory, all waves are absorbed and their vertical wavelengths become infinitely small at the critical level defined in Sect. 2.2; however, the waves are totally reflected at the height at which $m^{2}=0$. This height is called the turning level (Fritts and Alexander, 2003). The intrinsic phase speed at the turning level is given by

$\hat{c}_{\mathrm{h}}^{2}=\frac{N^{2}+\frac{f^{2}}{4 H^{2} k_{\mathrm{h}}^{2}}}{k_{\mathrm{h}}^{2}+\frac{1}{4 H^{2}}}$,

and, therefore, the vertically propagating gravity waves must have an intrinsic phase speed slower than that in Eq. (3). The intrinsic wave frequency at the turning level is equal to the buoyancy frequency under the condition $H \rightarrow \infty$ (i.e. the Boussinesq approximation). 


\section{Results}

Matsuda et al. (2014) extracted gravity waves with horizontal wavelengths $\left(\lambda_{\mathrm{h}} \equiv \frac{2 \pi}{k_{\mathrm{h}}}\right)$ of $5-100 \mathrm{~km}$ in their method. Thus, transmission diagrams for gravity waves with $\lambda_{h}=100,50$ and $10 \mathrm{~km}$ are considered in this study.

The buoyancy frequency squared, which is used in the subsequent analysis, is taken to be

$$
N^{2}=\left\{\begin{array}{cc}
2 \times 10^{-4}\left(\mathrm{~s}^{-2}\right) & 50 \mathrm{~km} \leq z \leq 90 \mathrm{~km} \text { (mesosphere) } \\
4 \times 10^{-4}\left(\mathrm{~s}^{-2}\right) & 10 \mathrm{~km} \leq z<50 \mathrm{~km} \text { (stratosphere) } \\
1 \times 10^{-4}\left(\mathrm{~s}^{-2}\right) & 0 \mathrm{~km} \leq z<10 \mathrm{~km} \text { (troposphere) }
\end{array}\right.
$$

In this treatment, it is assumed that the airglow emission layer exists at an altitude of $90 \mathrm{~km}$. The inertial frequency is taken to be the value at Syowa Station $\left(69^{\circ} \mathrm{S}\right.$; i.e. the inertial period is about $12.8 \mathrm{~h}$ ), but would be lower at lower latitudes.

When background horizontal winds and a horizontal wavelength of the wave are given, gravity wave transmissivity is obtained as follows: first, the intrinsic horizontal phase speed $\left(\hat{c}_{\mathrm{h}}\right)$ and vertical wavenumber squared $\left(\mathrm{m}^{2}\right)$ at each height are computed for specified ground-based horizontal phase speed and propagation direction using Eqs. (1) and (2), respectively. If $\hat{c}_{\mathrm{h}}$ or $\hat{c}_{\mathrm{h}} \mp \frac{f}{k_{\mathrm{h}}}$ change sign with height between starting height and $90 \mathrm{~km}$, the wave is considered to encounter the critical level of $\hat{c}_{\mathrm{h}}=0$ or $\hat{c}_{\mathrm{h}}= \pm \frac{f}{k_{\mathrm{h}}}$, respectively. On the other hand, if $m^{2}$ becomes zero or negative between starting height and $90 \mathrm{~km}$, the wave is considered to encounter the turning level. This calculation is performed for every phase speed and propagation direction, such that the transmission diagram is constructed.

Figure 1a-e show transmission diagrams for gravity waves with $\lambda_{h}=100 \mathrm{~km}$ in background horizontal winds with constant vertical shear (shown in Fig. 1f). The transmissivities for propagation to $90 \mathrm{~km}$ of the gravity waves emitted at $0 \mathrm{~km}$ (i.e. ground), $5 \mathrm{~km}$ (i.e. middle troposphere), $10 \mathrm{~km}$ (i.e. tropopause), $30 \mathrm{~km}$ (i.e. middle stratosphere) and $60 \mathrm{~km}$ (i.e. lower mesosphere) were computed. The black shaded regions represent the restricted vertical propagation of gravity waves due to the critical level filtering (outlined in Sect. 2.1). The restricted regions exhibit circular structures around the background horizontal winds that vary with height, similar to critical level blocking diagram results (Taylor et al., 1993; Medeiros et al., 2003; Dowdy et al., 2007). However, a region of allowed vertical propagation appears inside the region of restricted vertical propagation, especially in Fig. 1d and e. This region of allowed vertical propagation corresponds to those gravity waves for which the directions of the intrinsic and ground-based phase speeds are opposite, as mentioned in Sect. 2.1. For example, a gravity wave with an eastward ground-based phase speed of $50 \mathrm{~m} \mathrm{~s}^{-1}$ emitted at $60 \mathrm{~km}$ can propagate to the mesopause region, because its intrinsic phase speed is always negative between 60 and $90 \mathrm{~km}$ and it does not encounter the critical level (see Fig. 1e). Such gravity waves can exist in regions of strong background horizontal winds, such as the polar-night jet, and they have not been

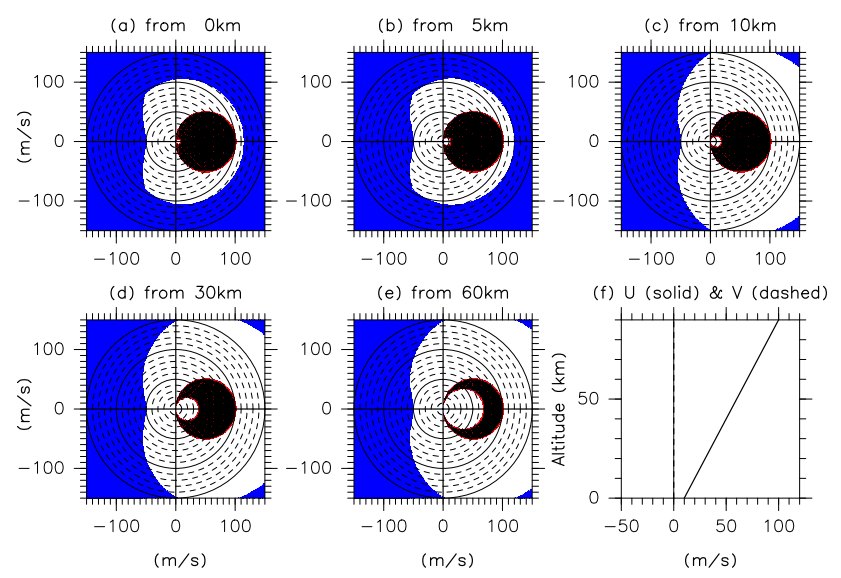

Figure 1. Gravity wave transmission diagrams for gravity waves propagating to the mesopause region with $\lambda_{\mathrm{h}}=100 \mathrm{~km}$ starting from (a) $0 \mathrm{~km}$, (b) $5 \mathrm{~km}$, (c) $10 \mathrm{~km}$, (d) $30 \mathrm{~km}$ and (e) $60 \mathrm{~km}$, and (f) vertical distributions of background (solid) zonal and (dashed) meridional winds. Black, red and blue shading represents restricted regions of gravity wave propagation due to critical level filtering, additional critical level filtering and turning level reflection, respectively.

considered in previous studies using critical level blocking diagrams.

The red shaded regions in Fig. 1a-e represent the restricted vertical propagation of gravity waves due to additional critical level filtering (outlined in Sect. 2.2). As $\frac{|f|}{k_{\mathrm{h}}} \approx 2 \mathrm{~m} \mathrm{~s}^{-1}$ for $\lambda_{\mathrm{h}}=100 \mathrm{~km}$ at Syowa Station, the region of critical level filtering is extended outward by about $2 \mathrm{~m} \mathrm{~s}^{-1}$ compared to the $\hat{c}_{\mathrm{h}}=0$ case. Since this effect becomes smaller for shorter horizontal wavelengths, its impact on gravity wave propagation is not expected to be significant for $\lambda_{\mathrm{h}} \leq 100 \mathrm{~km}$.

The blue shaded regions in Fig. 1a-e represent the restricted vertical propagation of gravity waves due to turning level reflection (outlined in Sect. 2.3). Turning level reflection occurs where the magnitude of the intrinsic phase speed of the wave is larger than the threshold value given in Eq. (3). Most gravity waves with ground-based horizontal phase speeds of $<50 \mathrm{~m} \mathrm{~s}^{-1}$ would be able to propagate vertically, except through the restricted region due to critical level filtering.

Figure 2a-e present transmission diagrams for gravity waves with $\lambda_{h}=50 \mathrm{~km}$. As critical level filtering only depends on the horizontal wavelength of the wave through $\frac{f}{k_{\mathrm{h}}}$ and this is small for this case, the black and red shaded regions are nearly the same as those in Fig. 1. However, the effects of the turning level reflection are distinctly larger than those in Fig. 1. Most gravity waves with westward groundbased phase speeds cannot propagate vertically, even if they are emitted in the stratosphere and mesosphere (Fig. 2d-e). Therefore, turning level reflection could play a significant role in the gravity wave power spectrum anisotropy around the mesopause obtained by airglow observations. The re- 


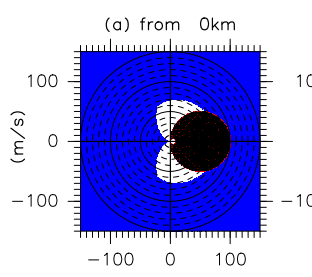

(b) from $5 \mathrm{~km}$

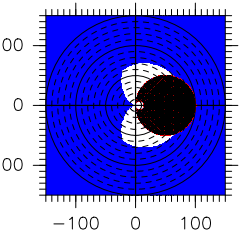

(d) from $30 \mathrm{~km}$

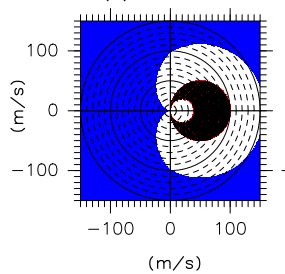

(e) from $60 \mathrm{~km}$

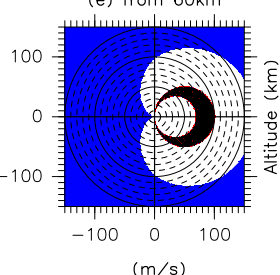

$(\mathrm{m} / \mathrm{s})$

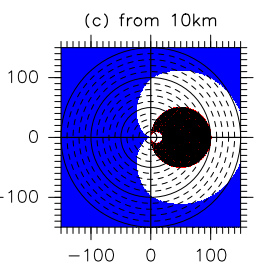

(f) U (solid) \& V (dashed)

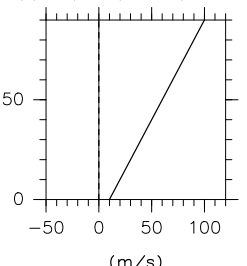

$(\mathrm{m} / \mathrm{s})$

Figure 2. Same as Fig. 1 except for $\lambda_{\mathrm{h}}=50 \mathrm{~km}$.

stricted region is larger for gravity waves emitted at 0 and $5 \mathrm{~km}$ than for those emitted at 10, 30 and $60 \mathrm{~km}$. This difference occurs because gravity waves propagating in regions of smaller buoyancy frequency such as in the troposphere have $\mathrm{m}^{2}$ values closer to zero (the turning level threshold) and are thus more prone to restriction in vertical propagation. This feature indicates that buoyancy frequency also has significant effect on gravity wave transmissivity.

Transmission diagrams for gravity waves with $\lambda_{h}=10 \mathrm{~km}$ are shown in Fig. 3. Almost all gravity waves are restricted to propagate vertically, even if they are emitted in the stratosphere and mesosphere. Therefore, it is difficult for gravity waves with short horizontal wavelengths, such as $10 \mathrm{~km}$, to propagate vertically in horizontal background winds that vary considerably with height (cf. Marks and Eckermann, 1995).

\section{Discussion}

The ground-based horizontal group speed for gravity waves with medium frequency (i.e. $N>>|\hat{\omega}|>>|f|$, where $\hat{\omega}$ is the intrinsic wave frequency) becomes the same as the ground-based horizontal phase speed (cf. Fritts and Alexander, 2003). If the period for vertical propagation of a wave from its source region to the mesopause is about $2 \mathrm{~h}$ (Stockwell and Lowe, 2001), then the horizontal propagation distance is at most a few hundred kilometres for gravity waves with $\left|c_{\mathrm{h}}\right| \leq 50 \mathrm{~m} \mathrm{~s}^{-1}$. Thus, the assumption that the horizontal variation of the background horizontal winds is negligible within the horizontal propagation distance of the wave is valid for background winds modified by large-scale waves, such as planetary waves and tides. However, the effects of large-scale gravity waves with horizontal wavelengths of several hundred kilometres or longer need to be considered carefully.
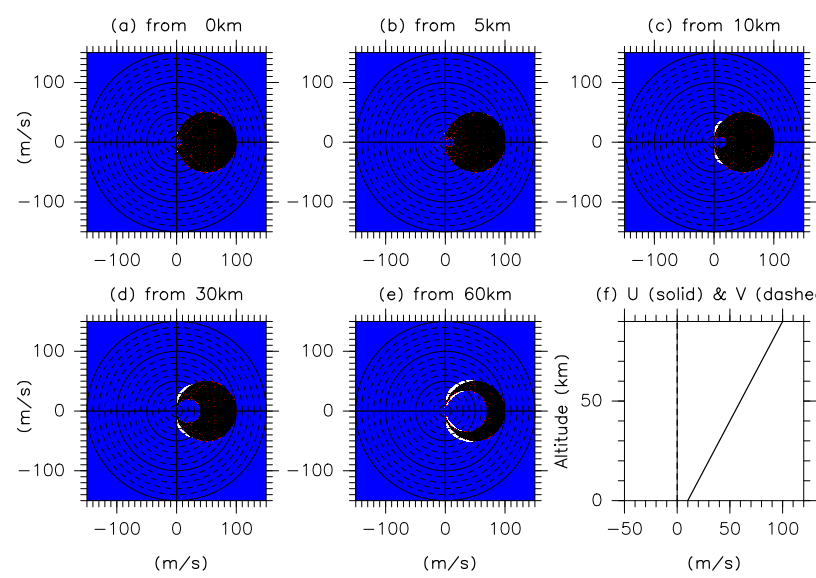

(e) from $60 \mathrm{~km}$

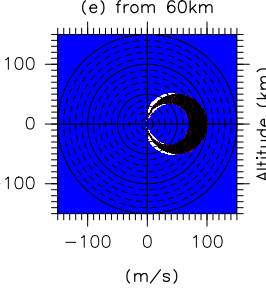

(f) $U$ (solid) \& V (dashed)

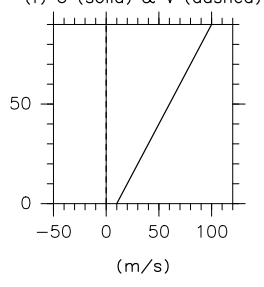

Figure 3. Same as Fig. 1 except for $\lambda_{h}=10 \mathrm{~km}$.

Gravity wave transmission diagrams in simple background horizontal winds with constant vertical shear showed the following:

- Gravity waves with $\lambda_{\mathrm{h}}=100 \mathrm{~km}$ and $\left|c_{\mathrm{h}}\right| \leq 50 \mathrm{~m} \mathrm{~s}^{-1}$ can propagate vertically, except for those filtered at the critical level.

- Gravity waves with $\lambda_{\mathrm{h}}=50 \mathrm{~km}$ can propagate vertically if the direction of their ground-based phase speed is roughly normal to the background horizontal winds.

- Gravity waves with $\lambda_{\mathrm{h}}=10 \mathrm{~km}$ cannot propagate vertically.

These features are true even in the real atmosphere above Syowa Station. As the vertical variations of the zonal wind are dominant over those of the meridional wind in winter, because of the polar-night jet, the gravity wave transmission diagram becomes similar to Figs. 1-3. Stratospheric westward winds become dominant, and the meridional wind is generally weak in summer. Gravity waves with westward and eastward ground-based phase speeds are restricted to propagate vertically by critical level filtering and turning level reflection, respectively, in summer.

In principle, the gravity wave transmission diagram can be applied to gravity waves with long horizontal wavelengths such as $\lambda_{\mathrm{h}}=1000 \mathrm{~km}$ and low intrinsic frequency (i.e. $|\hat{\omega}| \approx$ $|f|)$. While these gravity waves with low intrinsic frequency hardly encounter the turning level, additional critical level filtering significantly restricts their vertical propagation because of $\frac{|f|}{k_{\mathrm{h}}} \approx 20 \mathrm{~m} \mathrm{~s}^{-1}$ (not shown). However, an assumption of small horizontal propagation distance does not hold for these low-frequency gravity waves, because the ratio of vertical to horizontal group speed for these gravity waves is much smaller than those for high- and medium-frequency gravity waves (cf. Eq. 39 of Fritts and Alexander, 2003). Thus, the gravity wave transmission diagram should not be applied to the gravity waves with long horizontal wavelengths and low intrinsic frequency. 
A ray tracing theory has also been used to describe the horizontal and vertical propagation of gravity waves given varying background horizontal winds (Lighthill, 1978). The effects of planetary waves and tides on gravity wave propagation have been studied by Senf and Achatz (2011) and Kalisch et al. (2014) using ray tracing models. As the ray tracing of gravity waves provides accurate and realistic trajectories of the gravity wave packets, it should be explored if possible. However, ray tracing calculations require 3-D horizontal wind and temperature distributions throughout the calculation period. Generally, such data are not available near the mesopause, except in general circulation models. Thus, the gravity wave transmission diagram used in this study remains useful for the interpretation of gravity waves observed by airglow imagers, even if it assumes that the gravity waves only propagate vertically.

\section{Conclusions}

Gravity waves with relatively short horizontal wavelengths (i.e. $<100 \mathrm{~km}$ ) near the mesopause have been studied using airglow imagers. Recently, a new statistical method of obtaining the gravity wave power spectrum as a function of ground-based horizontal phase speed and propagation direction using airglow imager data was proposed (Matsuda et al., 2014). The gravity waves observed around the mesopause generally originate at lower altitudes, and these waves are affected by various processes such as critical level filtering, turning level reflection and turbulent and radiative dissipation during their upward propagation. A critical level blocking diagram, which considers the effects of critical level filtering, has been used to explain the gravity wave statistics observed by airglow imagers (Taylor et al., 1993; Stockwell and Lowe, 2001). However, the effects of turning level reflection have never been considered in such accounts. This report proposed a gravity wave transmission diagram that considers the effects of both critical level filtering and turning level reflection.

Transmission diagrams for gravity waves propagating to the mesopause region with different horizontal wavelengths and starting heights in simple background horizontal winds were presented in this study. In previous studies, critical level filtering has been taken into account for gravity waves with the same sign for their ground-based and intrinsic horizontal phase speeds. However, this study demonstrated that gravity waves with opposite signs for their ground-based and intrinsic horizontal phase speeds could reach the mesopause region when the gravity waves were emitted in the stratosphere and mesosphere. Although the effects of additional critical level filtering at $\hat{c}_{\mathrm{h}}= \pm \frac{f}{k_{\mathrm{h}}}$ were also evaluated, they were small for gravity waves with relatively short horizontal wavelengths observed by airglow imagers.

The effects of turning level reflection on gravity wave transmissivity were observed to depend considerably on the horizontal wavelength of the gravity waves. While most gravity waves with $\lambda_{\mathrm{h}}=100 \mathrm{~km}$ and $\left|c_{\mathrm{h}}\right| \leq 50 \mathrm{~m} \mathrm{~s}^{-1}$ could propagate vertically, except for those filtered at the critical level, almost all gravity waves with $\lambda_{\mathrm{h}}=10 \mathrm{~km}$ could not reach the mesopause region because of critical level filtering and turning level reflection. In the case of gravity waves with $\lambda_{\mathrm{h}}=50 \mathrm{~km}$, while gravity waves with eastward and westward ground-based phase speeds were not allowed to propagate vertically, because of critical level filtering and turning level reflection, respectively, gravity waves with southward and northward ground-based phase speeds could reach the mesopause region. These results indicate that, in addition to critical level filtering, turning level reflection is an important mechanism in determining gravity wave transmissivity.

Acknowledgements. The author thanks T. Nakamura, M. Tsutsumi, M. K. Ejiri, T. Nishiyama and T. S. Matsuda for their helpful comments. Figures were drawn using Dennou Club Library (DCL). The author would like to thank Enago (www.enago.jp) for the English language review.

The topical editor Christoph Jacobi thanks two anonymous referees for help in evaluating this paper.

\section{References}

Booker, J. R. and Bretherton, F. P.: The critical layer for internal gravity waves in a shear flow, J. Fluid Mech., 27, 513-539, 1967.

Dowdy, A. J., Vincent, R. A., Tsutsumi, M., Igarashi, K., Murayama, Y., Singer, W., and Murphy, D. J.: Polar mesosphere and lower thermosphere dynamics: 1. Mean wind and gravity wave climatologies, J. Geophys. Res., 112, D17104, doi:10.1029/2006JD008126, 2007.

Dunkerton, T. J. and Butchart, N.: Propagation and selective transmission of internal gravity waves in a sudden warming, J. Atmos. Sci., 41, 1443-1460, 1984.

Fritts, D. C. and Alexander, M. J.: Gravity wave dynamics and effects in the middle atmosphere, Rev. Geophys., 41, 1003, doi:10.1029/2001RG000106, 2003.

Hecht, J. H., Walterscheid, R. L., Hickey, M. P., and Franke, S. J.: Climatology and modeling of quasi-monochromatic atmospheric gravity waves observed over Urbana Illinois, J. Geophys. Res., 106, 5181-5195, doi:10.1029/2000JD900722, 2001.

Kalisch, S., Preusse, P., Ern, M., Eckermann, S. D., and Riese, M.: Differences in gravity wave drag between realistic oblique and assumed vertical propagation, J. Geophys. Res., 119, 1008110099, doi:10.1002/2014JD021779, 2014.

Lighthill, M. J.: Waves in Fluids, Cambridge Univ. Press, Cambridge, UK, 1978.

Marks, C. J. and Eckermann, S. D.: A three-dimensional nonhydrostatic ray-tracing model for gravity waves: Formulation and preliminary results for the middle atmosphere, J. Atmos. Sci., 52, 1959-1984, 1995.

Matsuda, T. S., Nakamura, T., Ejiri, M. K., Tsutsumi, M., and Shiokawa, K.: New statistical analysis of the horizontal phase velocity distribution of gravity waves observed by airglow imaging, J. Geophys. Res., 119, 9707-9718, doi:10.1002/2014JD021543, 2014. 
Medeiros, A. F., Taylor, M. J., Takahashi, H., Batista, P. P., and Gobbi, D.: An investigation of gravity wave activity in the lowlatitude upper mesosphere: Propagation direction and wind filtering, J. Geophys. Res., 108, 4411, doi:10.1029/2002JD002593, 2003.

Nakamura, T., Higashikawa, A., Tsuda, T., and Matsushita, Y.: Seasonal variations of gravity wave structures in $\mathrm{OH}$ airglow with a CCD imager at Shigaraki, Earth Planets Space, 51, 897-906, 1999.

Senf, F. and Achatz, U.: On the impact of middle-atmosphere thermal tides on the propagation and dissipation of gravity waves, J. Geophys. Res., 116, D24110, doi:10.1029/2011JD015794, 2011.

Stockwell, R. G. and Lowe, R. P.: Airglow imaging of gravity waves 2. Critical layer filtering, J. Geophys. Res., 106, 17205-17220, 2001.
Taylor, M. J., Ryan, E. H., Tuan, T. F., and Edwards, R.: Evidence of preferential directions for gravity wave propagation due to wind filtering in the middle atmosphere, J. Geophys. Res., 98, 60476057, doi:10.1029/92JA02604, 1993.

Thomas, L., Worthington, R. M., and McDonald, A. J.: Inertiagravity waves in the troposphere and lower stratosphere associated with a jet stream exit region, Ann. Geophys., 17, 115-121, 1999 , http://www.ann-geophys.net/17/115/1999/.

Whiteway, J. A. and Duck, T. J.: Evidence for critical level filtering of atmospheric gravity waves, Geophys. Res. Lett., 23, 145-148, 1996. 